\title{
Activation of Coeruleospinal Noradrenergic Inhibitory Controls during Withdrawal from Morphine in the Rat
}

\author{
Dana S. Rohde ${ }^{2,3}$ and Allan I. Basbaum ${ }^{1,2,3}$ \\ Program in Biomedical Sciences, Departments of ${ }^{1}$ Anatomy and ${ }^{2}$ Physiology and ${ }^{3} W$. M. Keck Foundation Center for \\ Integrative Neuroscience, University of California at San Francisco, San Francisco, California 94143
}

\begin{abstract}
We previously reported that withdrawal from morphine induces the expression of Fos, a marker of neuronal activity, in spinal cord neurons, particularly in laminae I and II of the superficial dorsal horn, and that the magnitude of Fos expression is increased in rats with a midthoracic spinal transection. We suggested that loss of withdrawal-associated increases in descending inhibitory controls that arise in the brainstem underlie the increased Fos expression after spinal transection. Here, we addressed the origin of the supraspinal inhibition. We injected rats intracerebroventricularly with saline or anti-dopamine- $\beta$-hydroxylase-saporin, a toxin that destroys noradrenergic neurons of the locus coeruleus. Eleven days later, we implanted rats with morphine or placebo pellets, and after $4 \mathrm{~d}$, we precipitated withdrawal with naltrexone. One hour later, the rats were killed, their brains and spinal cords were removed, and transverse sections of
\end{abstract}

the brains and spinal cords were immunoreacted with an antibody to Fos.

In placebo-pelleted rats, the toxin injection did not alter behavior and did not induce expression of the Fos protein. However, compared with saline-injected withdrawing rats, the toxin-treated rats that underwent withdrawal demonstrated an intense withdrawal behavior rarely seen in the absence of toxin, namely forepaw fluttering. The rats also had significantly increased Fos-like immunoreactivity in all laminae of the cervical cord and in laminae I and II and the ventral horn of the lumbar cord. No differences were recorded in the sacral cord. We conclude that the effects of spinal transection in rats that withdraw from morphine in part reflect a loss of coeruleospinal noradrenergic inhibitory controls.

Key words: descending inhibition; Fos; locus coeruleus; morphine withdrawal; saporin; spinal cord; tolerance
Chronic administration of opioids produces a state of tolerance in which subsequent doses of opioids are less effective, resulting in, among other things, reduced antinociception. Recent studies suggest that opioid tolerance results from the development of a compensatory response in the adenylyl cyclase system in neurons that express the opioid receptor or in neural circuits in which those neurons participate (Sharma et al., 1975; Nestler and Tallman, 1988; Nestler, 1992). This compensatory response, which brings neuronal firing rate and cAMP levels to control levels, counteracts the reduction of cAMP produced by morphine. Opioid-tolerant neurons are considered to be in a state of "latent hyperexcitability" (Fry et al., 1980), which is revealed when the opioid is displaced from the opioid receptor, leaving the upregulated cAMP pathway unopposed. This is manifest by increased adenylyl cyclase activity and behavioral signs of withdrawal.

Another manifestation of this compensatory response is increased neuronal activity and Fos expression in locus coeruleus (LC) neurons of withdrawing rats (Duman et al., 1988; Nestler and Tallman, 1988; Guitart and Nestler, 1989; Nestler et al., 1989; Hayward et al., 1990). These authors hypothesized that this increased activity drives the withdrawal syndrome. In related studies, we described extensive expression of the Fos protein in neurons of the superficial dorsal horn of the spinal cord of withdrawing rats and hypothesized that a development of latent

\footnotetext{
Received Dec. 1, 1997; revised March 18, 1998; accepted March 23, 1998. This study was supported by National Institutes of Health Grant DA 08377. Correspondence should be addressed to Dr. Dana S. Rohde, Department of Anatomy, Box 0452, University of California, San Francisco, 513 Parnassus Avenue, Room S-1334, San Francisco, CA 94143-0452.

Copyright (C) 1998 Society for Neuroscience $\quad 0270-6474 / 98 / 184393-10 \$ 05.00 / 0$
}

hyperexcitability or sensitization in these neurons underlies withdrawal-induced hyperalgesia (Rohde et al., 1996).

To identify factors that regulate the increased spinal cord Fos-like immunoreactivity (Fos-LI) that occurs during withdrawal, we studied the effect of spinal transection at the T3 segment. In these rats, we found increased Fos-LI of the lumbar cord dorsal and ventral horns of withdrawing rats compared with unoperated withdrawing rats. Because descending inhibitory controls that regulate the firing of dorsal horn neurons are eliminated by spinal cord transection, we hypothesized that the increased expression of the Fos protein reflects a loss of inhibitory controls that are normally activated during systemic withdrawal and/or loss of tonic inhibitory control during the development of morphine tolerance (Rohde et al., 1997b).

The LC is one of the major supraspinal sources of inhibitory controls (Hodge et al., 1980; Jones and Gebhart, 1986). Because LC neurons are activated during withdrawal (Aghajanian, 1978; Hayward et al., 1990), we hypothesized that the inhibitory contribution of LC neurons is augmented during withdrawal and that transection eliminates this, resulting in the increased Fos-LI that we observed. This view of the LC contrasts with the prevailing one, noted above, namely that withdrawal-induced activity in the LC drives and possibly initiates some of the behavioral manifestations of the opiate withdrawal syndrome (Redmond and Krystal, 1984; Rasmussen and Aghajanian, 1989; Rasmussen et al., 1990; Nestler, 1992).

The objective of the present study was to specifically assess the contribution of the LC to behavior and to spinal cord neuronal activity during precipitated withdrawal from morphine. To this end, we studied the pattern of Fos-LI during withdrawal in rats 

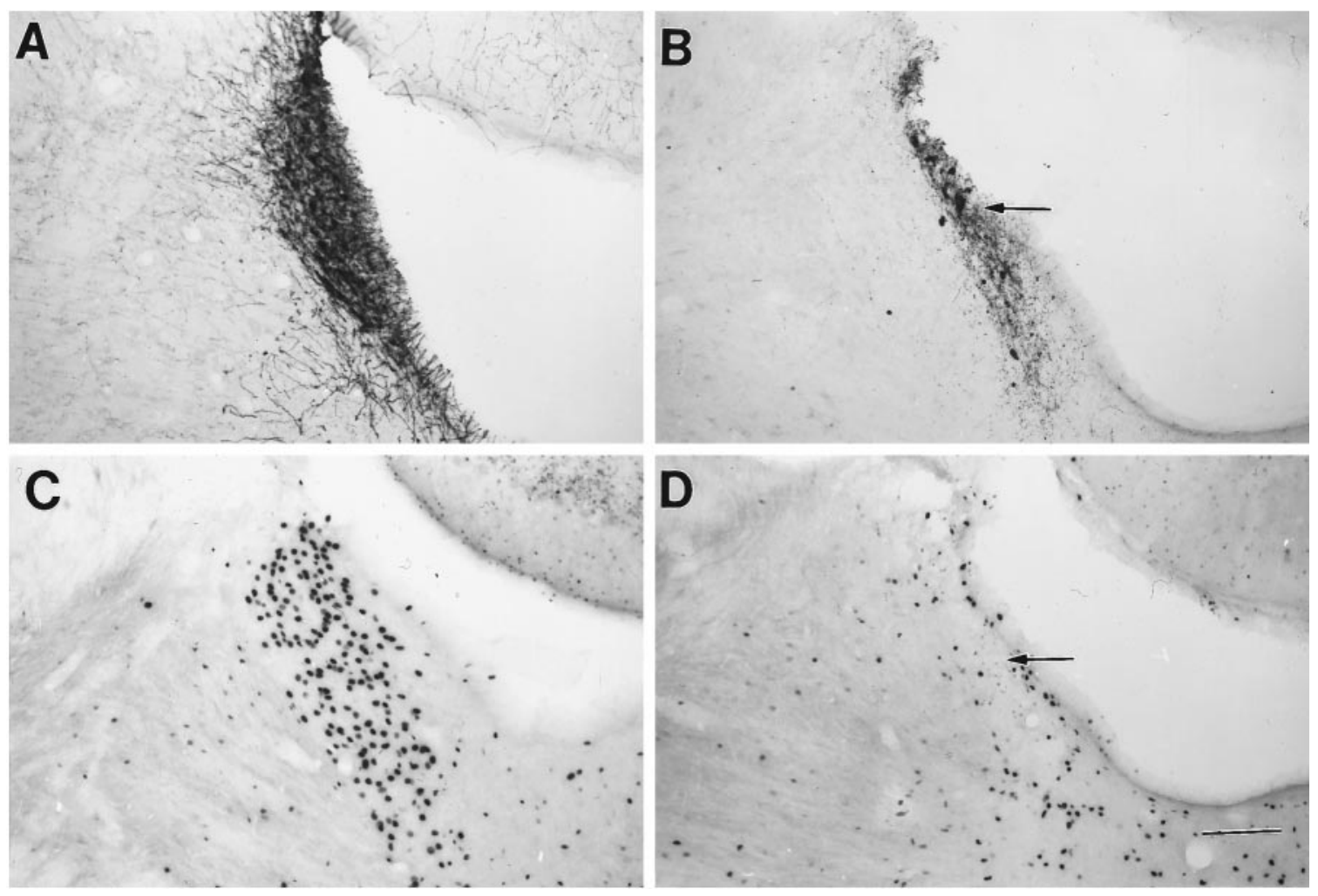

Figure 1. $\mathrm{D} \beta \mathrm{H}$ and Fos immunoreactivity in the LC. $A, \mathrm{D} \beta \mathrm{H}$ immunocytochemistry in the LC of a saline-injected withdrawing rat; $B$, D $\beta \mathrm{H}$ immunocytochemistry in the LC of a toxin-injected withdrawing rat; $C$, Fos-LI in the LC of a saline-injected withdrawing rat; and $D$, Fos-LI in the LC of a toxin-injected withdrawing rat. Note the decrease in $\mathrm{D} \beta \mathrm{H}$ immunoreactivity and decrease in Fos-LI in the toxin-treated rats (arrows). Scale bar, $200 \mu \mathrm{m}$.

that were pretreated with saline or anti-dopamine- $\beta$-hydroxylase $(\mathrm{D} \beta \mathrm{H})$-saporin, a toxin that selectively destroys noradrenergic neurons of the LC and its projections. We demonstrate that destruction of the LC releases an intense withdrawal behavior, namely increased forepaw fluttering, and increases Fos-LI in the spinal cord dorsal and ventral horns. We conclude that withdrawal-induced activity of spinally projecting LC neurons normally inhibits spinal cord neuronal activity.

\section{MATERIALS AND METHODS}

Experiments were performed on male Sprague Dawley rats (BantinKingman, Fremont, CA) with the approval of the Institutional Animal Care and Use Committee at University of California at San Francisco. The rats were housed two per cage on a $12 \mathrm{hr}$ light/dark cycle with ad libitum access to food and water. The rats were randomly assigned into four different treatment groups. One group was injected with the anti$\mathrm{D} \beta \mathrm{H}$-saporin immunotoxin into the lateral ventricle, and then the group was subdivided into placebo-pelleted $(n=4)$ and morphine-pelleted $(n=14)$ rats. Another group was injected with saline into the lateral ventricle and then also subdivided into placebo-pelleted $(n=4)$ and morphine-pelleted $(n=12)$ groups. The toxin was kindly provided by Dr. Ron Wiley (Veteran's Administration Medical Center, Nashville, TN).

The "immunotoxin" is composed of an antibody to $\mathrm{D} \beta \mathrm{H}$, the biosynthetic enzyme for norepinephrine, that is coupled to saporin (Wrenn et al., 1996), a ribosomal inactivating protein. The immunotoxin binds the membrane-bound subunit of $\mathrm{D} \beta \mathrm{H}$ that is exposed during exocytosis of norepinephrine. After the toxin is endocytosed, it undergoes retrograde axonal transport to the neuronal cell body, in which it arrests protein synthesis.

Rats weighed $240-260 \mathrm{gm}$ on the day of surgery. We anesthetized the rats with $0.15 \mathrm{cc}$ of xylazine and $0.3 \mathrm{cc}$ of ketamine and placed them in a stereotaxic apparatus. A hole was drilled into the lateral ventricle using the following coordinates from bregma: anteroposterior, -1.0 ; mediolateral, -1.5 ; and from skull: dorsoventral, -4.3 . Next, we lowered a 10 $\mu$ l Hamilton syringe into the lateral ventricle and slowly injected either toxin $(5.25 \mu \mathrm{g}$ toxin and $5.0 \mu \mathrm{l}$ saline) or saline $(5.0 \mu \mathrm{l})$ over $2 \mathrm{~min}$. Gelfoam was placed over the hole in the skull, and wound clips were used to close the skin.

Eleven days later, we implanted pellets subcutaneously $(75 \mathrm{mg}$ morphine sulfate or placebo pellet; kindly provided by National Institute on Drug Abuse, Rockville, MD) according to the following schedule: one on day 1 , two on day 2 , and three on day 3 . We previously demonstrated that this schedule induces tolerance to and dependence on morphine in rats (Rohde et al., 1997b). On day four (2 weeks after the surgery), we injected all of the rats with $10 \mathrm{mg} / \mathrm{kg}$ naltrexone, subcutaneously into the nape of the neck. The naltrexone was diluted in $800 \mu \mathrm{l}$ of physiological saline.

We monitored the animals for signs of withdrawal as follows. The rats were placed in a $12 \times 12 \times 12$ inch Plexiglas box, which was covered to prevent the rats from escaping. Tape divided the floor into four 6 square inch quadrants. The rats were acclimated to the box for $1 \mathrm{hr}$ and then observed for $10 \mathrm{~min}$ before and for $60 \mathrm{~min}$ after the injection of naltrexone. The observer had no knowledge of the treatments. Withdrawal behavior was divided into counted signs and checked signs. The behavior was monitored in 10 min bins. Behavioral activity was quantified by four counted signs: (1) number of events of forepaw flutters; (2) number of jumps; (3) number of times the rat crosses the taped line; and (4) number of wet-dog shakes. Forepaw flutters are rapid stereotypic movements of the forepaws. Jumps were scored when all four paws left the ground. The number of teeth-chattering events was also recorded. Checked signs included salivation, ptosis, diarrhea, chewing, teeth chattering, and chromodacchyorrhea. Weight loss was also monitored; the rats were weighed immediately before being placed in the box and $1 \mathrm{hr}$ after the naltrexone injection.

The rats were then deeply anesthetized with pentobarbital $(200 \mathrm{mg} / \mathrm{kg}$, i.p.) followed immediately by intracardiac perfusion with $100 \mathrm{ml}$ of $0.1 \mathrm{M}$ PBS, $\mathrm{pH} 7.4$, followed by $500-600 \mathrm{ml}$ of $10 \%$ formalin in $0.1 \mathrm{M}$ phosphate buffer (PB). The brains and spinal cords were removed $1 \mathrm{hr}$ later and post-fixed in $10 \%$ formalin for an additional $3 \mathrm{hr}$. After cryoprotection overnight in $30 \%$ sucrose in $0.1 \mathrm{M} \mathrm{PB}, 50 \mu \mathrm{m}$ frozen sections were cut in the transverse plane (spinal cord) or coronal plane (brain) and collected in $0.05 \mathrm{M}$ PBS for immunocytochemical analysis. 

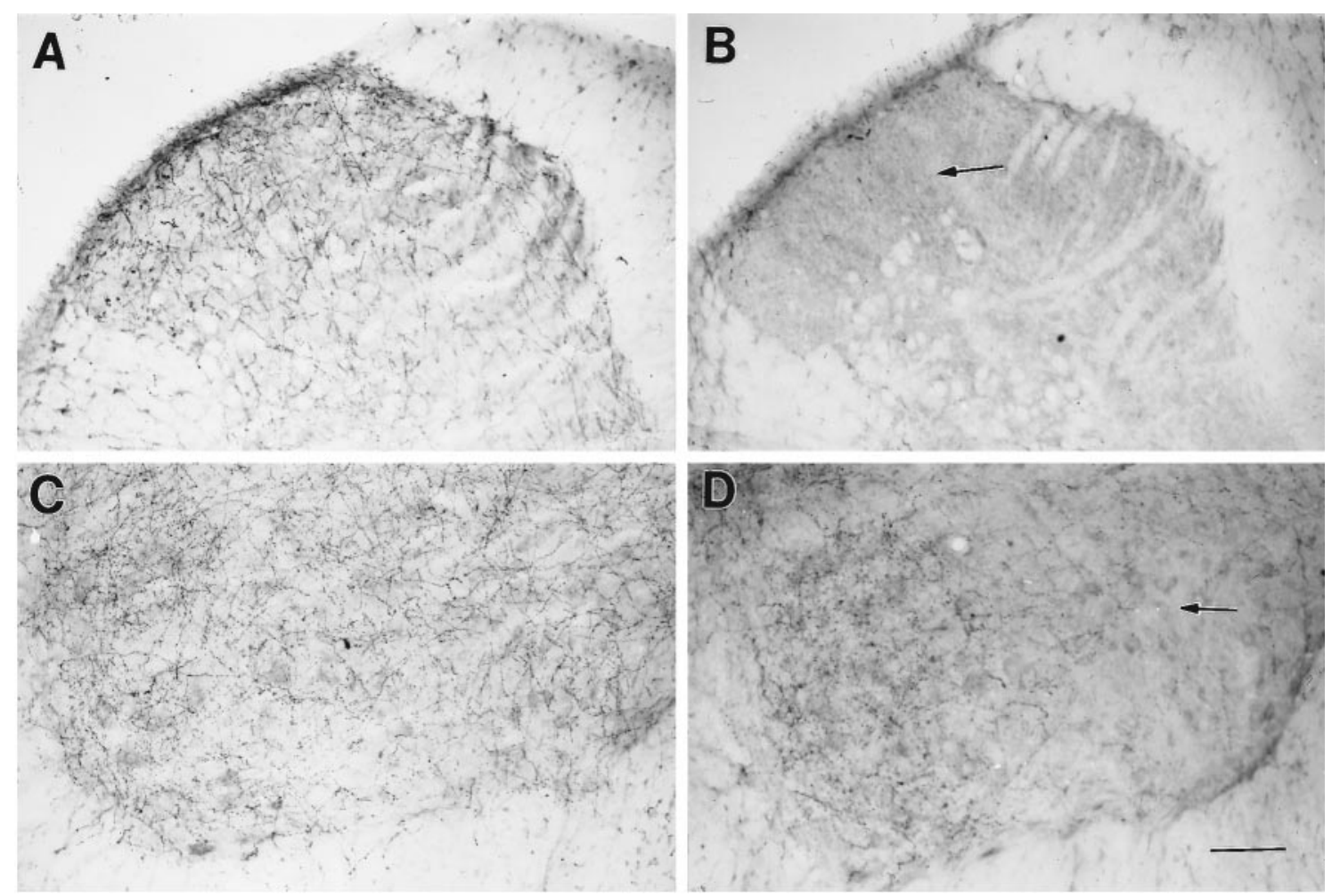

Figure 2. $\mathrm{D} \beta \mathrm{H}$ immunoreactivity in the spinal cord. $A$, Lumbar dorsal horn of a saline-injected withdrawing rat; $B$, lumbar dorsal horn of a toxin-injected withdrawing rat; $C$, lumbar ventral horn of a saline-injected withdrawing rat; and $D$, lumbar ventral horn of a toxin-injected withdrawing rat. Note the complete loss of $\mathrm{D} \beta \mathrm{H}$ immunoreactivity in the dorsal horn of the toxin-treated rat $(B)$ and the partial loss of $\mathrm{D} \beta \mathrm{H}$ immunoreactivity in the ventral horn of the toxin-treated rat $(D)$ (arrows). Scale bar, $100 \mu \mathrm{m}$.

Immunocytochemistry. The sections were immunostained for Fos, $\mathrm{D} \beta \mathrm{H}$, or tyrosine hydroxylase $(\mathrm{TH})$ by the avidin-biotin-peroxidase method of Hsu et al. (1981), as described below. We washed the sections with a buffer solution of $0.05 \mathrm{M}$ Tris PBS with $1 \%$ normal goat serum and $0.3 \%$ Triton X-100 and then incubated them for $1 \mathrm{hr}$ at room temperature in a blocking solution of $3 \%$ normal goat serum in $0.05 \mathrm{M}$ Tris PBS with $0.3 \%$ Triton $\mathrm{X}-100$. The blocking solution was removed from the tissue, and the sections were incubated overnight at room temperature in the primary antiserum in the same buffer. The Fos antibody (kindly provided by Dr. D. Slamon, University of California at Los Angeles) was diluted 1:30,000, the anti-D $\beta \mathrm{H}$ antibody (EugeneTech, Ridgefield Park, NJ) was diluted 1:5000, and the anti-TH antibody (EugeneTech) was diluted 1:1000. The primary antibody was removed, and the sections were washed and then incubated in biotinylated goat anti-rabbit IgG and avidin-biotin-peroxidase complex (Elite kit; Vector Laboratories, Burlingame, $\mathrm{CA}$ ). To visualize the $\mathrm{D} \beta \mathrm{H}$ or $\mathrm{TH}$ immunoreactivity as a brown reaction product, we used a diaminobenzidine glucose oxidase reaction after a protocol adapted from Llewellyn-Smith and Minson (1992). To visualize the Fos-LI as a black reaction product we used a nickeldiaminobenzidine reaction. After the immunoreaction, brain and spinal cord sections were mounted and coverslipped.

Quantification. To quantitate the numbers of Fos-LI neurons, the cervical (C7 and C8), lumbar (L4 and L5), and sacral (S1) sections were photographed at $4 \times$ (low) power with Eastman Kodak (Rochester, NY) technical pan film on a Nikon Microphot-FXA microscope. To analyze the LC, we first examined the sections under dark field to identify the appropriate levels for counting. We selected sections that contained the superior cerebellar peduncle and the motor nucleus of $\mathrm{V}$. We then switched to bright-field and photographed the sections. The film was developed with HC110 Dilution E developer, stopped with Kodak stop bath, and fixed with Kodak rapid fixative. For the spinal cord analysis, we divided individual sections of the cervical and lumbar cord into four regions: (1) the superficial laminae (laminae I, II outer, and II inner), (2) the nucleus proprius (laminae III and IV), (3) the neck of the dorsal horn (laminae V and VI), and (4) the ventral horn (laminae V II, V III, IX, and $\mathrm{X})$. The sacral cord was divided into six regions as described previously: (1) laminae I and II, (2) laminae III and IV, (3) laminae V and VI, (4) the sacral parasympathetic nucleus, (5) lamina $X$, and (6) the ventral horn (Rohde et al., 1997a). Fos-LI neurons were identified by a person blinded to the treatments. Up to six spinal cord or brain sections were examined per rat and the number of Fos-LI cells in each of these regions was counted and averaged so that each animal had a mean value for regional Fos-LI. We first performed a two-way ANOVA for total Fos for intracerebroventricular pretreatment (toxin or saline injection) and drug treatment (morphine and naltrexone or placebo and naltrexone). The numbers of labeled cells per $50 \mu \mathrm{m}$ section were compared using two-way ANOVAs for treatment and laminae and within laminae one-way ANOVAs for treatment. Fisher's protected least significant difference was used for post hoc comparisons. Each behavioral measure was analyzed separately with a $t$ test.

\section{RESULTS}

\section{Verification of toxin-induced destruction of the LC}

Neither morphine nor placebo pellets altered the pattern or density of $\mathrm{D} \beta \mathrm{H}$ immunoreactivity in the LC of rats that received saline instead of toxin. However, we noted a marked depletion in $\mathrm{D} \beta \mathrm{H}$ immunoreactivity in the LC (Fig. 1), cerebellum, and spinal cord dorsal horn of the toxin-treated rats (Fig. 2). Because there was an almost complete loss of staining in the toxin-treated rats, we did not attempt to quantitate the differences. By contrast, the $\mathrm{D} \beta \mathrm{H}$ immunoreactivity in $\mathrm{A} 5, \mathrm{~A} 7$, and the thoracic intermediolateral cell columns did not differ between the groups (Fig. 3).

Comparable results were recorded with $\mathrm{TH}$ antisera (Fig. 4). As for $\mathrm{D} \beta \mathrm{H}$, in the absence of toxin, neither morphine nor placebo pellets altered the pattern or density of TH immunoreactivity, but we noted a marked depletion in $\mathrm{TH}$ immunoreactivity in the LC of the toxin-treated rats compared with the saline-injected rats. Importantly, in the substantia nigra, a dopamine-rich region, we 
Figure 3. $\mathrm{D} \beta \mathrm{H}$ immunoreactivity in the $\mathrm{A} 5$ cell group and thoracic spinal cord. $A$, A5 cell group of a saline-injected withdrawing rat; $B, \mathrm{~A} 5$ cell group of a toxin-injected withdrawing rat; $C$, thoracic spinal cord of a saline-injected withdrawing rat; and $D$, thoracic spinal cord of a toxin-injected withdrawing rat. Note the A5 cell group (small arrows) and the intermediolateral cell columns of the thoracic cord (arrowheads) in the toxin-treated rats appear to retain their $\mathrm{D} \beta \mathrm{H}$ staining. Note the extensive loss of $\mathrm{D} \beta \mathrm{H}$ immunoreactivity in the dorsal horn of the toxin-treated rats (large arrow). Scale bar, $200 \mu \mathrm{m}$.
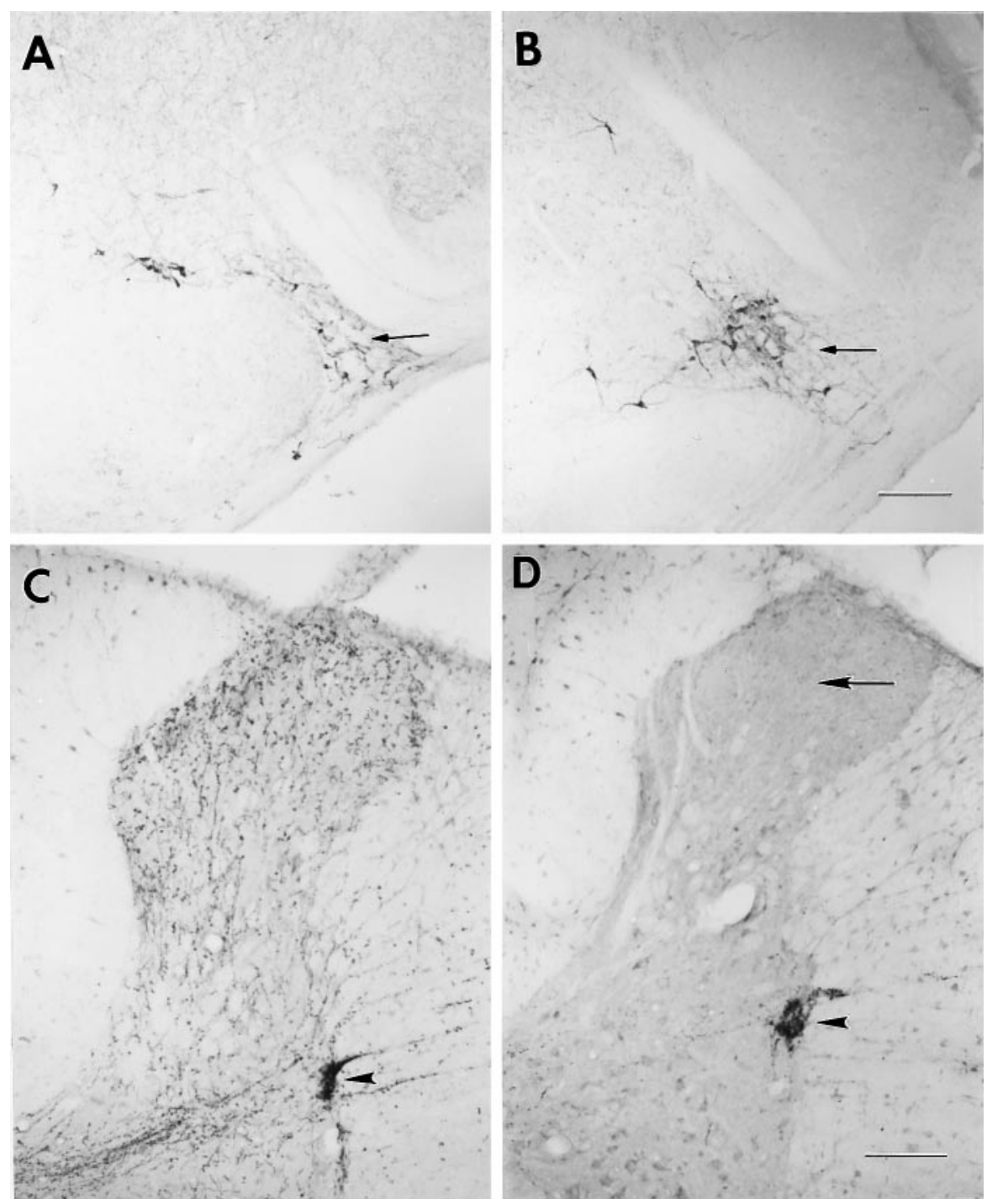

did not detect any loss of TH immunoreactivity in the toxintreated rats compared with the control rats.

Fos expression in the LC of nonwithdrawing rats is normally very low (Hayward et al., 1990). Consistent with this, we did not detect Fos-labeled cells in the LC of the placebo-pelleted rats that received naltrexone (Fig. 5). Furthermore, the toxin treatment did not significantly alter Fos-LI in the LC in placebo-pelleted rats compared with the placebo-pelleted rats injected intracerebroventricularly with saline as demonstrated by two-way ANOVA for total Fos using pretreatment and drug treatment. By contrast, the withdrawing rats demonstrated significantly increased Fos-LI in the LC compared with nonwithdrawing rats, which agrees with a previous study (Hayward et al., 1990). Finally, the toxin-treated withdrawing rats demonstrated significantly less Fos-LI (67\%) in the LC compared with the saline-treated withdrawing rats (Figs. 1, 5). In fact, the Fos-LI in the LC of the toxin-treated withdrawing rats did not differ significantly from that of the placebopelleted groups.

\section{Withdrawal behavior}

The toxin treatment did not alter the normal weight gain of the rats, and all rats appeared healthy throughout the 2 week period.
Neither the saline-injected nor the toxin-injected rats that received placebo pellets demonstrated unusual behavior, and, as expected, they showed no signs of withdrawal behavior after naltrexone. By contrast to these placebo-pelleted rats, the morphine-pelleted rats that received an intracerebroventricular saline or saporin injection demonstrated profound signs of withdrawal including salivation, ptosis, diarrhea, chewing, teeth chattering, and chromodacryorrhea (Table 1).

The most striking difference between the two morphinepelleted withdrawing groups (the intracerebroventricularly saline-injected and intracerebroventricularly saporin-injected withdrawing rats) was the presence of bouts of intense forepaw fluttering in the saporin-injected but not the saline-injected group; the difference between the groups was highly significant $(p<0.01)$ (Fig. 6). Although both the checked signs of withdrawal (Table 1) and most of the counted signs of withdrawal hyperactivity were greater in the morphine-pelleted, saporintreated rats than in the morhpine-pelleted, saline-treated rats (Fig. 6), the differences were not statistically significant. Although both morphine-pelleted groups had significant weight loss after withdrawal, there was no difference in the weight loss (expressed 

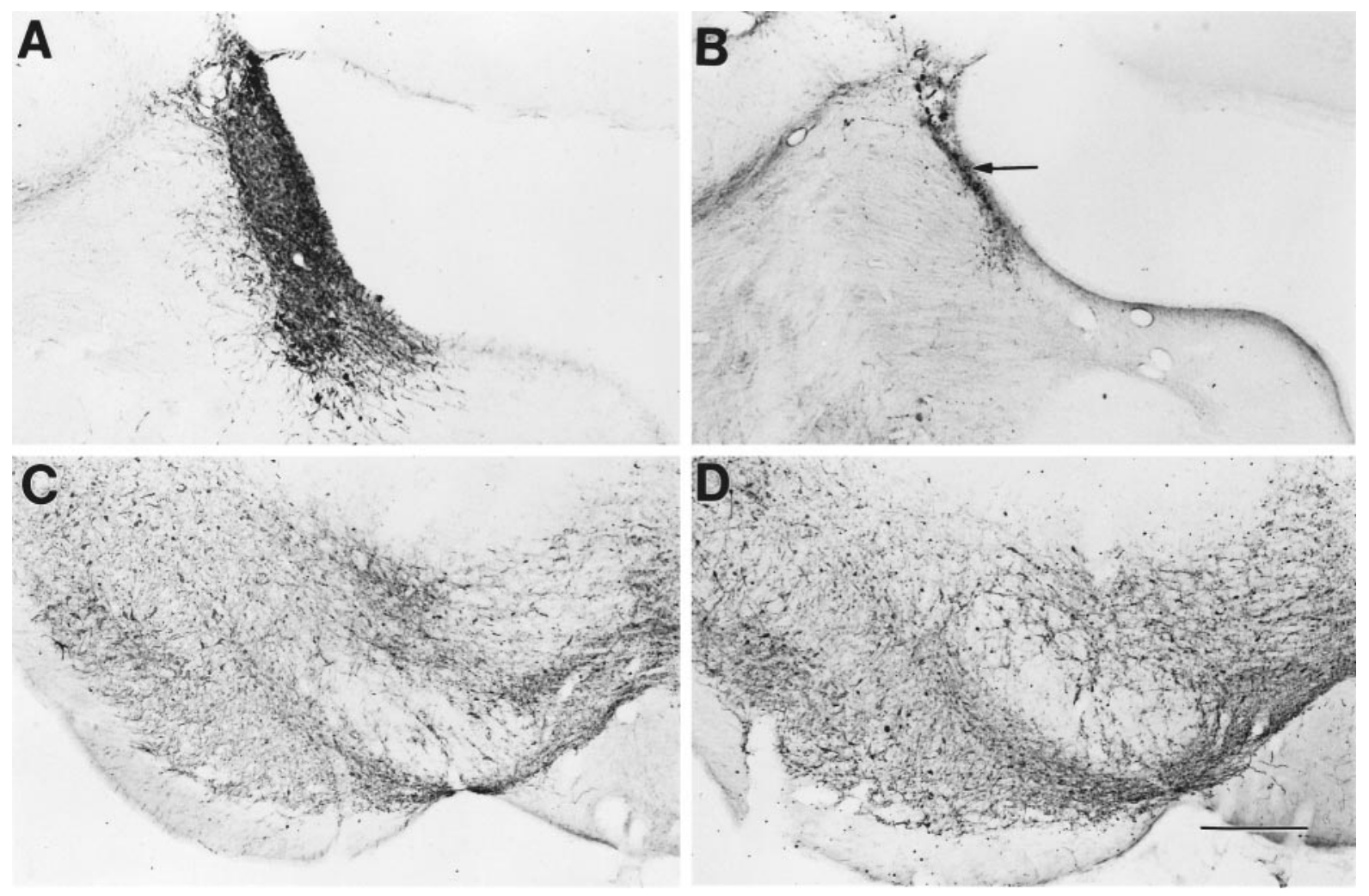

Figure 4. TH immunoreactivity in the LC and substantia nigra. $A$, TH immunoreactivity in the LC of a saline-injected withdrawing rat; $B$, TH immunoreactivity in the $\mathrm{LC}$ of a saporin-injected withdrawing rat; $C$, TH immunoreactivity in the substantia nigra of a saline-injected withdrawing rat; $D$, TH immunoreactivity in the substantia nigra of a saporin-injected withdrawing rat. Note the profound reduction of TH immunoreactivity (arrow) in the LC of the saporin-treated rat $(B)$; by contrast TH immunoreactivity in the substantia nigra of the saporin-treated rat $(D)$ was not changed. Scale bar, $250 \mu \mathrm{m}$.

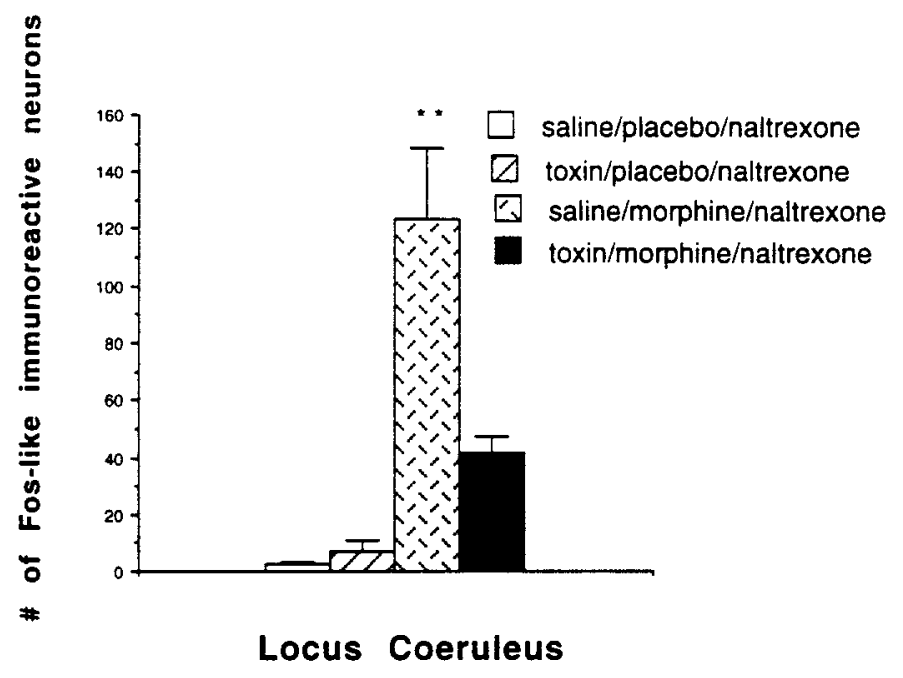

Figure 5. Number of Fos-labeled neurons per $50 \mu \mathrm{m}$ section through the LC. The LC of the saline-pretreated withdrawing rats had significantly more Fos-LI than did the nonwithdrawing rats or the toxin-treated withdrawing rats $(* * p<0.01)$.

as a percentage of prewithdrawal weight) between these two groups (Fig. 7).

\section{Fos-like immunoreactivity in the spinal cord}

We previously reported that withdrawal from morphine induces significant increases in Fos expression in the spinal cord. These increases were demonstrated in the lumbar dorsal horn (Rohde et al., 1996) and in the sacral dorsal horn, lamina X, and sacral parasympathetic nuclei (Rohde et al., 1997a). Importantly, naltrexone injection in placebo-pelleted rats does not induce Fos expression in the spinal cord.

Toxin treatment, by itself, did not significantly alter Fos-LI in any regions of the spinal cord in placebo-pelleted rats. Compared with saline-treated rats, however, withdrawal-induced total Fos expression was significantly increased in the spinal cord after toxin treatment (Figs. 8, 9). In the cervical cord, the toxin-treated withdrawing rats demonstrated significantly increased Fos-LI in all laminae (increase vs saline group; $68 \%$ in laminae I and II, $86 \%$ in laminae III and IV, $109 \%$ in laminae V and VI, and $153 \%$ in the ventral horn). The toxin-treated withdrawing rats also demonstrated significantly increased Fos-LI in the superficial dorsal horn laminae I and II (65\% increase vs saline group) and the ventral horn $(83 \%)$ of the lumbar cord. Finally, in the sacral cord, we did not record any differences between the toxin-treated and the saline-treated withdrawing rats (Figs. 8, 10).

\section{DISCUSSION}

In a previous study we reported that midthoracic spinal cord transection increases the magnitude of Fos expression in the lumbar spinal cord of withdrawing rats compared with unoperated rats (Rohde et al., 1997b). From these data, we hypothesized that there is a supraspinal source of inhibition that is activated during morphine withdrawal. In the present study, we demonstrate an intense withdrawal behavior that is rarely seen, namely increased forepaw fluttering as well as increased Fos-LI in the 
Table 1. Number of animals in each treatment group in which the indicated withdrawal sign was present

\begin{tabular}{lccclll} 
Treatment & Salivation & Ptosis & Diarrhea & Chewing & Chattering & Chromo \\
\hline Saline, MN $(n=12)$ & 11 & 11 & 8 & 7 & 6 & 6 \\
Saporin, MN $(n=14)$ & 13 & 12 & 11 & 6 & 7 & 5 \\
Saline, PN $(n=4)$ & 0 & 0 & 0 & 0 & 0 & 0 \\
Saporin, PN $(n=4)$ & 0 & 0 & 0 & 0 & 0 & 0 \\
\hline
\end{tabular}

MN, Morphine/naltrexone; PN, placebo/naltrexone; Chromo, chromodaccryorrhea.

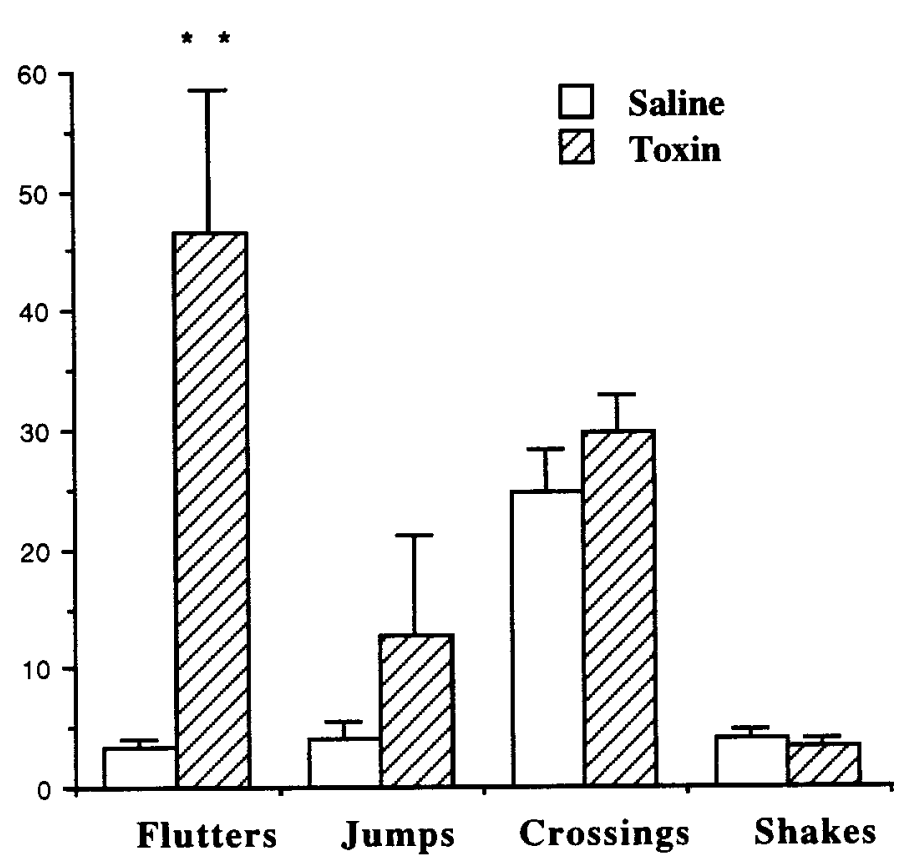

Figure 6. Behavioral activity in the saline and saporin-treated withdrawing groups. Forepaw fluttering was significantly increased in the saporintreated withdrawing group as compared with the saline-treated withdrawing group. There were no significant differences in the other behaviors $(* * p<0.01)$.

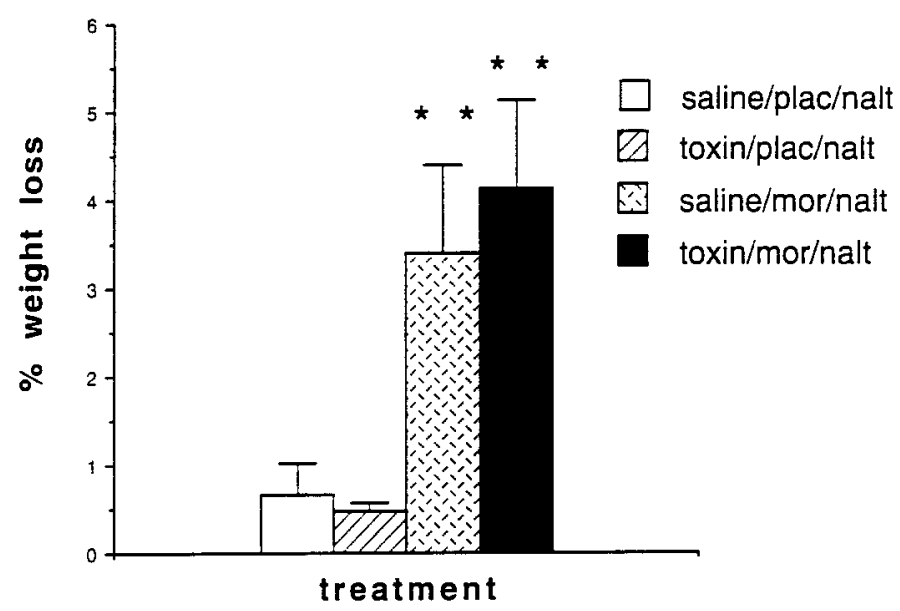

Figure 7. Percentage weight loss in the four treatment groups. Withdrawing rats lost significantly more weight than did the nonwithdrawing rats, but the toxin treatment did not alter the withdrawal-induced weight loss $(* * p<0.01)$.

spinal cord of withdrawing rats that have a neurotoxin-induced depletion of the noradrenergic cell bodies of the LC and of the descending noradrenergic innervation of the spinal cord dorsal horn. Because activity of LC neurons is associated with decreased firing of dorsal horn neurons (Hodge et al., 1980; Jones and Gebhart, 1986), our results suggest that the LC normally inhibits spinal cord neurons during withdrawal. It follows that the increased activity of LC neurons produced by systemic withdrawal must counteract the increased activity of spinal neurons and the withdrawal syndrome to which these neurons contribute.

\section{Specificity of the anti-D $\boldsymbol{\beta H}$-saporin immunotoxin}

Our conclusion concerning the contribution of the LC in part derives from the ability of the immunotoxin treatment to selectively target and destroy the LC and its projections, while sparing serotonergic, dopaminergic, and noncoerulear noradrenergic nuclei and their projections. In fact, our results with the toxin are the same as previously described (Wrenn et al., 1996). We confirmed the profound loss of $\mathrm{D} \beta \mathrm{H}$ immunoreactivity in the $\mathrm{LC}$ and importantly, we also found decreased withdrawal-induced Fos expression in the LC of toxin-treated rats, indicating that the destruction was of cell bodies in the LC. The toxin did not destroy the other major noradrenergic brainstem nuclei, the A5 and A7 cell groups, and the density of $\mathrm{D} \beta \mathrm{H}$ staining in a major target of these nuclei, the intermediolateral cell column, was unchanged. Although some studies reported an LC projection to the ventral horn of the spinal cord (Proudfit and Clark, 1991), others did not (Fritschy et al., 1987). Because we only found a small decrease of $\mathrm{D} \beta \mathrm{H}$ staining in the ventral horn, it appears that the LC projects to the ventral horn, but that it is not the predominant source of the noradrenergic innervation of this region. Importantly, the placebo-pelleted group that was injected intracerebroventricularly with saporin and later with naltrexone did not demonstrate any unusual behaviors. The critical control group establishes that the toxin itself does not cause any seizure-like behaviors or side effects that could interfere with our interpretation of the changes in withdrawal behavior.

\section{Withdrawal behavior and Fos-LI}

The toxin-treated withdrawing rats were considerably more active than the saline-treated withdrawing rats. In addition, only in the toxin-treated withdrawing rats did we detect an increase in Fos-LI in the superficial dorsal and ventral horns of the lumbar cord compared with the nontreated withdrawing rats. These are the same regions of the cord in which we detected increased Fos-LI after spinal transection (Rohde et al., 1997b). Although the extent to which the LC projects to both the dorsal and ventral horns is unclear (see above, Fritschy et al., 1987; Proudfit and Clark, 1991), the Fos results taken together with the pattern of loss of $\mathrm{D} \beta \mathrm{H}$ immunoreactivity suggest that the LC projects primarily to the dorsal horn but also to the ventral horn, and that it normally exerts an inhibitory control during withdrawal.

It was only in the cervical cord of the toxin-treated withdrawing rats that we saw increased Fos-LI in all laminae, including laminae III and IV. The latter region receives inputs from large- 


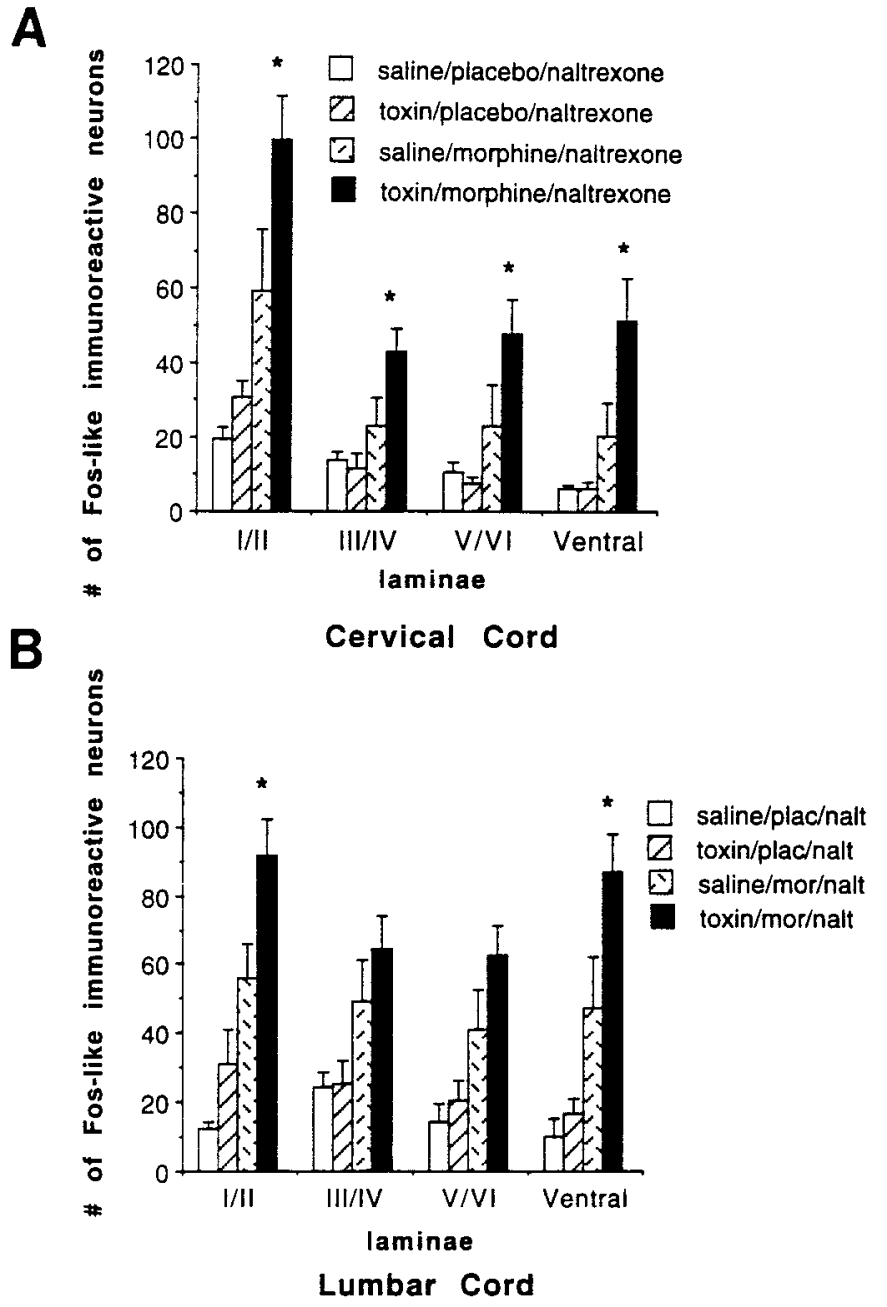

C

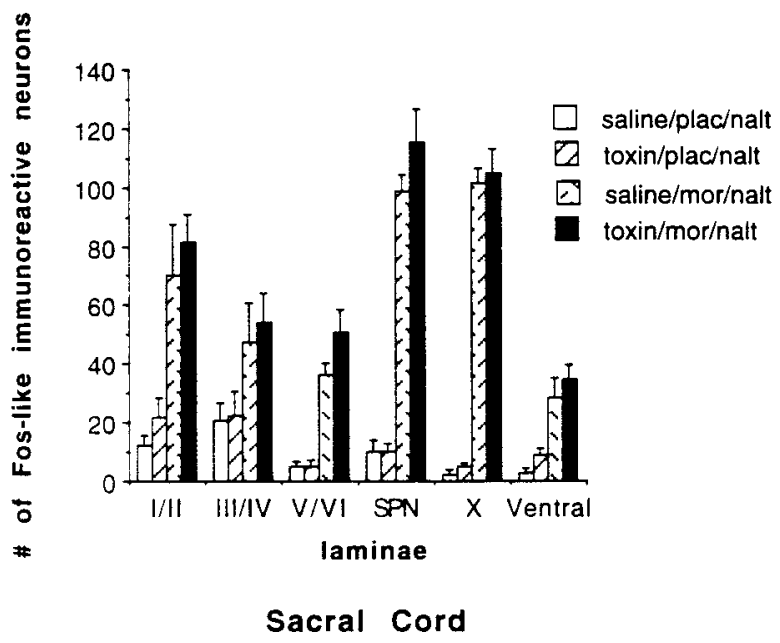

Figure 8. Number of Fos-like immunoreactive neurons per $50 \mu \mathrm{m} \mathrm{sec-}$ tion of the spinal cord. $A$, Cervical cord; $B$, lumbar cord; and $C$, sacral cord. The toxin-treated rats demonstrated significantly increased Fos-LI in all cervical laminae compared with the saline-injected withdrawing rats. We recorded significantly increased Fos-LI in the lumbar superficial dorsal horn and ventral horn of the toxin-treated withdrawing rats compared with the saline-injected withdrawing rats. There was no difference in the sacral cord between the toxin- and saline-injected withdrawing rats. Fos expression remained extremely low at all levels of the spinal cord in the placebo-pelleted rats that were injected with saline or toxin. $(p<0.05)$. diameter afferents that respond to non-noxious stimuli. We previously demonstrated that movement of the limbs induces Fos expression in laminae III and IV of the cervical or lumbar cord (Presley et al., 1990; Jasmin et al., 1994; Rohde et al., 1996). Thus, it is likely that the increased Fos-LI in this region is caused by the significantly increased forepaw fluttering. Because we only rarely observed forepaw fluttering in the saline-injected withdrawing rats and because forepaw tremor has been documented in mice withdrawing from morphine (Majeed et al., 1994), we do not believe that the fluttering is a behavior unique to saporin-treated withdrawing rats. Rather we suggest that this behavior is normally inhibited by withdrawal-induced increased activity of LC neurons that project to the spinal cord. We hypothesize that destruction of the LC blocks that inhibitory activity and unmasks this behavior. Interestingly, comparable behavior has been described in rats withdrawing from cannabinoids (Tsou et al., 1995). The authors proposed that alterations in circuits in the basal ganglia underlie these responses.

Because parasympathetic preganglionic neurons in the sacral cord are a target of descending LC projections (Westlund and Coulter, 1980; Westlund et al., 1983) and are under noradrenergic inhibitory control (Ryall and deGroat, 1972), we expected to see an effect of the toxin on withdrawal-induced diarrhea, weight loss, and sacral cord Fos-LI. The fact that we did not suggests that withdrawal-induced increased Fos expression in the sacral spinal cord is not influenced by descending inhibitory controls that originate in the LC and that LC innervation of the spinal cord does not alter withdrawal-induced gastrointestinal motility. This conclusion is, in fact, consistent with our previous demonstration that withdrawal-induced diarrhea and sacral cord Fos expression is largely driven by enhanced peripheral activity (Rohde et al., 1997a).

\section{Contribution of the LC to withdrawal from morphine}

Electrophysiological (Aghajanian, 1978) and immunocytochemical (Hayward et al., 1990) studies suggest that the LC is activated during withdrawal from morphine. Consistent with this formulation, clonidine, an $\alpha 2$ adrenergic receptor agonist that reduces the firing of LC neurons through an inhibitory autoreceptor, suppresses withdrawal-induced increases in $c$-fos mRNA in the LC (Rasmussen et al., 1995) and suppresses withdrawal-induced increases in the turnover of norepinephrine in the cerebral cortex (Zigun et al., 1981). We also observed activation of the LC during withdrawal from morphine. Because the LC inhibits the firing of spinal cord neurons (Hodge et al., 1980; Jones and Gebhart, 1986), it follows that activation of the LC would attenuate the increased firing that occurs in the setting of withdrawal.

Although this conclusion agrees with studies that used 6-OHDA to destroy catecholamines (Elchisak and Rosecrans, 1979; Friedler et al., 1972), it is at odds with several studies that concluded that withdrawal-induced LC activity accounts for (i.e., drives) the behavioral signs of withdrawal (Rasmussen et al., 1990; Koob et al., 1992; Maldonado and Koob, 1993). The latter conclusion is based largely on the use of clonidine, which suppresses some but not all behavioral signs of withdrawal (Charney et al., 1982; Jasinski et al., 1985; Taylor et al., 1988). Importantly, because clonidine has actions in the spinal cord (Franz et al., 1982; Yasuoka and Yaksh, 1983), and because opioid withdrawal has been demonstrated at the spinal level (Delander and Takemori, 1983; Delander et al., 1984; Rohde et al., 1996), conclusions based on results produced by systemic injection of clonidine may not be correct. On the other hand, it is difficult to reconcile the 

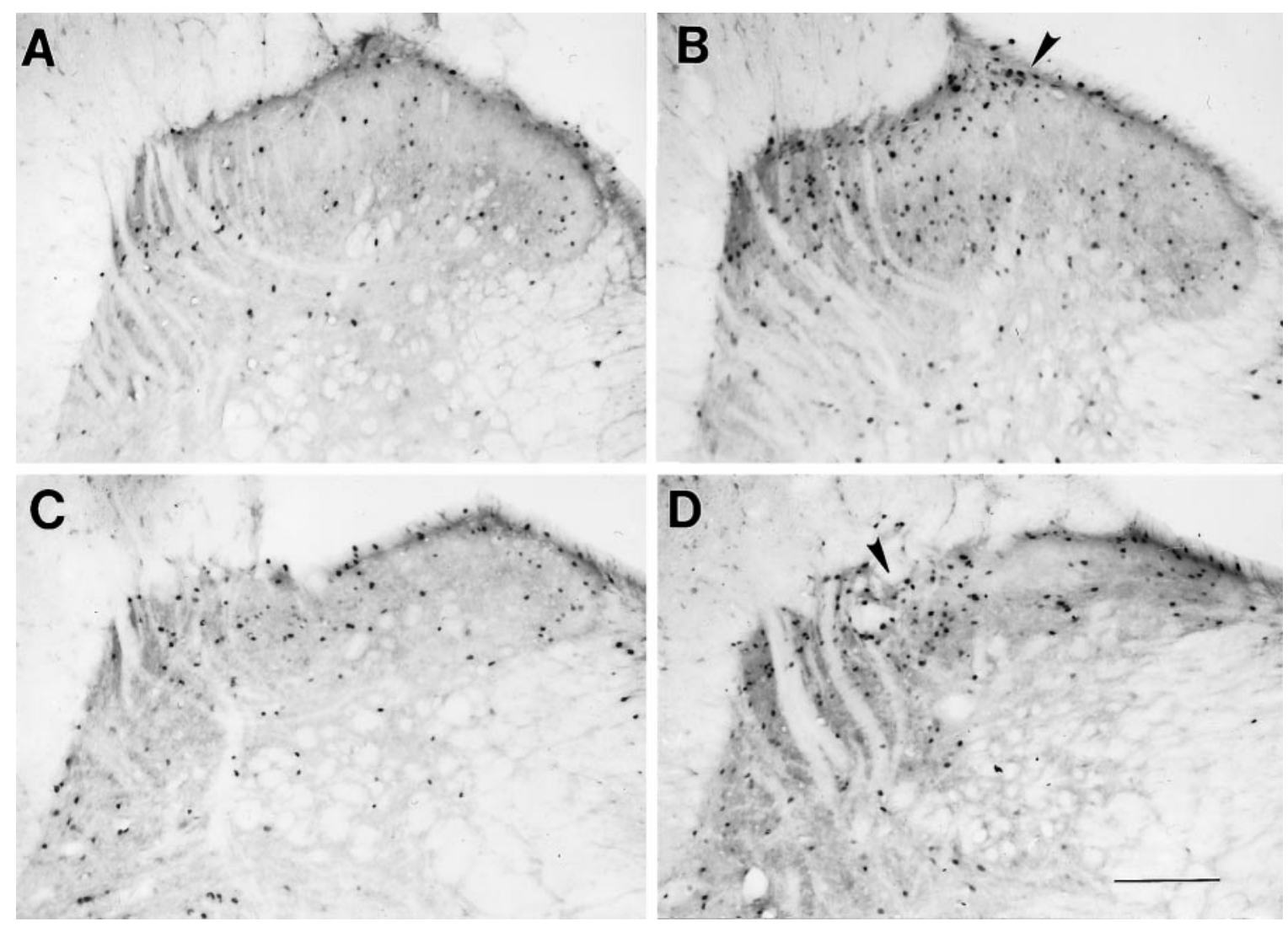

Figure 9. Withdrawal-induced Fos-LI in the spinal cord dorsal horn. $A$, Cervical dorsal horn of a saline-injected withdrawing rat; $B$, cervical dorsal horn of a toxin-injected withdrawing rat; $C$, lumbar dorsal horn of a saline-injected withdrawing rat; and $D$, lumbar dorsal horn of a toxin-injected withdrawing rat. Note the increase in Fos-LI in laminae I and II of the toxin-treated withdrawing rats $(B, D$; arrowheads) and the increase in Fos-LI in laminae III and IV in the cervical spinal cord of the toxin-treated withdrawing rat $(B)$. Scale bar, $200 \mu \mathrm{m}$.

fact that clonidine injected directly into the $\mathrm{LC}$ also reduces withdrawal signs (Aghajanian, 1978; Taylor et al., 1988) and that an opioid antagonist injected directly into the LC produces an intense withdrawal (Koob et al., 1992). One possibility is that there are subpopulations of neurons within the LC. Those which project to the spinal cord may, when activated, attenuate the magnitude of withdrawal; the ascending component may be facilitatory.

It is, of course, also possible that withdrawal induces LC activity, but that LC activity does not induce withdrawal behavior. In fact, some studies suggest that there is no relationship between LC activity and withdrawal behavior. For example, Chieng and Christie (1995) reported that the behavioral withdrawal syndrome is not altered in rats pretreated with saline or DSP4, a toxin that destroys LC nerve terminals (Fritschy et al., 1990). In that behavioral study, however, D $\beta \mathrm{H}$ immunoreactivity was only tested in the cortex, hippocampus, and cerebellum, not the spinal cord. Furthermore, noradrenaline concentrations only decreased by $50 \%$, leaving substantial residual noradrenaline to activate the signs of withdrawal. We suggest that the enhanced withdrawal and increased Fos expression that we observed reflect the near complete loss of the coeruleospinal noradrenergic innervation.

As noted by Simonato (1996), although tolerance and withdrawal-like phenomena can be studied at the molecular and cellular levels and in specific nervous system sites, the circuitry of the neurons that express the opioid receptor as well as the neurons that are synaptically connected to the opioid- bearing neurons must also be considered. Indeed, the effects of chronic morphine in an in vitro system or in an isolated brain site may not accurately model the effects seen in vivo. These differences underscore the importance of studying circuit function in the whole animal when integrated responses, such as withdrawal from morphine, are being evaluated.

\section{Summary}

In summary, by selective destruction of the catecholaminergic outflow of the LC, we provide strong evidence that activation of the LC during naltrexone-precipitated withdrawal from morphine dampens the withdrawal syndrome. Not only did the neurotoxic destruction of the LC increase withdrawal-induced forepaw fluttering, but it also increased the magnitude of spinal cord Fos expression, a marker of neuronal activity. We conclude that the effects of spinal transection in rats that withdraw from morphine in part reflect a loss of coeruleospinal noradrenergic inhibitory controls. Our results emphasize that the withdrawal syndrome produced by systemic injection of an antagonist reflects the integrated action of excitatory and inhibitory circuits that interconnect disparate regions of the brain and spinal cord. The possibility that the development of tolerance is influenced both by adaptive changes in these networks and by cellular responses to persistent opioids (i.e., decoupling of opioid receptors and inhibitory G-proteins) must be considered. 

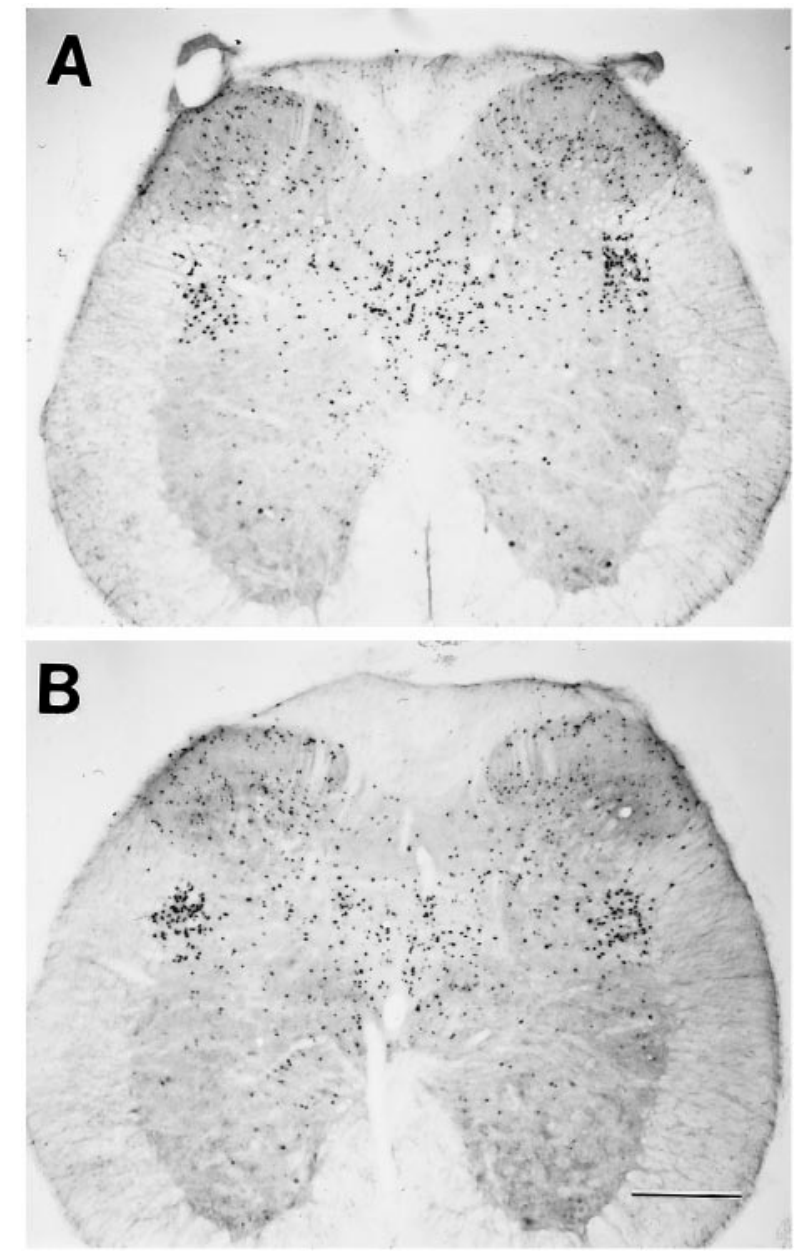

Figure 10. Fos-LI in the sacral spinal cord. $A$, Sacral spinal cord of a saline-injected withdrawing rat; and $B$, sacral spinal cord of a toxininjected withdrawing rat. There were no differences in Fos-LI between these two groups. Scale bar, $500 \mu \mathrm{m}$.

\section{REFERENCES}

Aghajanian GK (1978) Tolerance of locus coeruleus neurones to morphine and suppression of withdrawal response by clonidine. Nature 276:186-188.

Charney DS, Riordan CE, Kleber HD, Murburg M, Braverman P, Sternberg DE, Heninger GR, Redmond DE (1982) Clonidine and naltrexone: a safe, effective, and rapid treatment of abrupt withdrawal from methadone therapy. Arch Gen Psychiatry 39:1327-1332.

Chieng B, Christie MJ (1995) Lesions to terminals of noradrenergic locus coeruleus neurones do not inhibit opiate withdrawal behaviour in rats. Neurosci Lett 186:37-40.

Delander GE, Takemori AE (1983) Spinal antagonism of tolerance and dependence induced by systemically administered morphine. Eur J Pharmacol 94:35-42.

Delander GE, Portoghese PS, Takemori AE (1984) Role of spinal mu opioid receptors in the development of morphine tolerance and dependence. J Pharmacol Exp Ther 231:91-96.

Duman RS, Tallman JF, Nestler EJ (1988) Acute and chronic opiateregulation of adenylate cyclase in brain: specific effects in locus coeruleus. J Pharmacol Exp Ther 246:1033-1039.

Elchisak MA, Rosecrans JA (1979) Development of morphine tolerance and physical dependence in rats depleted of brain catecholamines by 6-hydroxydopamine. Neuropharmacology 18:175-182.

Franz DN, Hare DB, McCloskey KL (1982) Spinal sympathetic neurons: possible sites of opiate-withdrawal suppression by clonidine. Science 215:1643-1645.

Friedler G, Bhargava HN, Quock R, Way EL (1972) The effect of 6-hydroxydopamine on morphine tolerance and physical dependence. J Pharmacol Exp Ther 183:49-55.

Fritschy J-M, Lyons WE, Mullen CA, Kosofsky BE, Molliver ME, Grzanna R (1987) Distribution of locus coeruleus axons in the rat spinal cord: a combined anterograde transport and immunohistochemical study. Brain Res 437:176-180.

Fritschy J-M, Geffard M, Grzanna R (1990) The response of noradrenergic axons to systemically administered DSP-4 in the rat: an immunohistochemical study using antibodies to noradrenaline and dopamine$\beta$-hydroxylase. J Chem Neuroanat 3:309-321.

Fry JP, Herz A, Zieglgansberger W (1980) A demonstration of naloxone-precipitated opiate withdrawal on single neurones in the morphine-tolerant/dependent rat brain. Br J Pharmacol 68:585-592.

Guitart X, Nestler EJ (1989) Identification of morphine- and cyclic AMP-regulated phosphoproteins (MARPPs) in the locus ceruleus and other regions of rat brain: regulation by acute and chronic morphine. J Neurosci 9:4371-4387.

Hayward MD, Duman RS, Nestler EJ (1990) Induction of the c-fos proto-oncogene during opiate withdrawal in the locus coeruleus and other regions of rat brain. Brain Res 525:256-266.

Hodge Jr C J, Apkarian AV, Stevens R, Volgelsang G, Wisnicki HJ (1980) Locus coeruleus modulation of dorsal horn unit responses to cutaneous stimulation. Brain Res 204:415-420.

Hsu S, Raine L, Fanger H (1981) A comparative study of the peroxidaseantiperoxidase method and an avidin-biotin complex method for studying polypeptide hormones with radioimmunoassay antibodies. Am J Clin Pathol 75:734-738.

Jasinski DR, Johnson RE, Kocher TR (1985) Clonidine in morphine withdrawal: differential effects on signs and symptoms. Arch Gen Psychiatry 42:1063-1066.

Jasmin L, Gogas KR, Ahlgren SC, Levine JD, Basbaum AI (1994) Walking evokes a distinctive pattern of Fos-like immunoreactivity in the caudal brainstem and spinal cord of the rat. Neuroscience 58:275-286.

Jones SL, Gebhart GF (1986) Quantitative characterization of coeruleospinal inhibition of nociceptive transmission in the rat. J Neurophysiol 56:1397-1410.

Koob GF, Maldonado R, Stinus L (1992) Neural substrates of opiate withdrawal. Trends Neurosci 15:186-191.

Llewellyn-Smith IJ, Minson JB (1992) Complete penetration of antibodies into vibratome sections after glutaraldehyde fixation and ethanol treatment: Light and electron microscopy for neuropeptides. J Histochem Cytochem 40:1741-1749.

Majeed NH, Przewlocka B, Machelska H, Przewlocki R (1994) Inhibition of nitric oxide synthase attenuates the development of morphine tolerance and dependence in mice. Neuropharmacology 33:189-192.

Maldonado R, Koob GF (1993) Destruction of the locus coeruleus decreases physical signs of opiate withdrawal. Brain Res 605:128-138.

Nestler EJ (1992) Molecular mechanisms of drug addiction. J. Neurosci 12:2439-2450.

Nestler EJ, Tallman JF (1988) Chronic morphine treatment increases cyclic AMP-dependent protein kinase activity in the rat locus coeruleus. Mol Pharmacol 33:127-132.

Nestler EJ, Erdos JJ, Terwilliger R, Duman RS, Tallman JF (1989) Regulation of G-proteins by chronic morphine in the rat locus coeruleus. Brain Res 476:230-239.

Presley RW, Menétrey D, Levine JD, Basbaum AI (1990) Systemic morphine suppresses noxious stimulus-evoked Fos protein-like immunoreactivity in the rat spinal cord. J Neurosci 10:323-335.

Proudfit HK, Clark FM (1991) The projections of locus coeruleus neurons to the spinal cord. In: Progress in brain research, Vol 88, The neurobiology of the locus coeruleus (Barnes CD, Pompeiano O, eds), pp 123-141. Amsterdam: Elsevier Science.

Rasmussen K, Aghajanian GK (1989) Withdrawal-induced activation of locus coeruleus neurons in opiate-dependent rats: attenuation by lesions of the nucleus paragigantocellularis. Brain Res 505:346-350.

Rasmussen K, Beitner-Johnson DB, Krystal JH, Aghajanian GK, Nestler EJ (1990) Opiate withdrawal and the rat locus coeruleus: behavioral, electrophysiological, and biochemical correlates. J Neurosci 10:2308-2317.

Rasmussen K, Brodsky M, Inturrisi CE (1995) NMDA antagonists and clonidine block c-fos expression during morphine withdrawal. Synapse 20:68-74.

Redmond Jr DE, Krystal JH (1984) Multiple mechanisms of withdrawal from opioid drugs. Annu Rev Neurosci 7:443-478. 
Rohde DS, Detweiler DJ, Basbaum AI (1996) Spinal cord mechanisms of opioid tolerance and dependence: Fos-like immunoreactivity increases in subpopulations of spinal cord neurons during withdrawal. Neuroscience 72:233-242.

Rohde DS, McKay WR, Abbadie C, Basbaum AI (1997a) Contribution of sacral spinal cord neurons to the autonomic and somatic consequences of withdrawal from morphine in the rat. Brain Res 745:83-95.

Rohde DS, McKay WR, Chang D, Abbadie C, Basbaum AI (1997b) The contribution of supraspinal, peripheral and intrinsic spinal circuits to the pattern and magnitude of Fos-like immunoreactivity in the lumbar spinal cord of the rat withdrawing from morphine. Neuroscience 80:599-612.

Ryall RW, deGroat WC (1972) The microelectrophoretic administration of noradrenaline, 5-hydroxytryptamine, acetylcholine and glycine to sacral parasympathetic preganglionic neurones. Brain Res 37:345-347.

Sharma SK, Klee WA, Nirenberg M (1975) Dual regulation of adenylate cyclase accounts for narcotic dependence and tolerance. Proc Natl Acad Sci USA 72:3092-3096.

Simonato M (1996) The neurochemistry of morphine addiction in the neocortex. Trends Pharmacol 17:410-415.

Taylor JR, Elsworth JD, Garcia EJ, Grant SJ, Roth RH, Redmond Jr DE (1988) Clonidine infusions into the locus coeruleus attenuate behav- ioral and neurochemical changes associated with naloxone-precipitated withdrawal. Psychopharmacology 96:121-134.

Tsou K, Patrick SL, Walker JM (1995) Physical withdrawal in rats tolerant to 9-tetrahydrocannabinol precipitated by a cannabinoid receptor antagonist. Eur J Pharmacol 280:R13-R15.

Westlund KN, Coulter JD (1980) Descending projections of the locus coeruleus and subcoeruleus/medial parabrachial nuclei in the monkey: axonal transport studies and dopamine-beta-hydroxylase immunocytochemistry. Brain Res 2:235-264.

Westlund KN, Bowker RM, Ziegler MG, Coulter JD (1983) Noradrenergic projections to the spinal cord of the rat. Brain Res 263:15-31.

Wrenn CC, Picklo MJ, Lappi DA, Robertson D, Wiley RG (1996) Central noradrenergic lesioning using anti-D $\beta \mathrm{H}$-saporin: anatomical findings. Brain Res 740:175-184.

Yasuoka S, Yaksh TL (1983) Effects of nociceptive threshold and blood pressure of intrathecally administered morphine and alpha-adrenergic agonists. Neuropharmacology 22:309-315.

Zigun JR, Bannon MJ, Roth RH (1981) Comparison of two alphaadrenergic agonists (clonidine and guanfacine) on norepinephrine turnover in the cortex of rats during morphine abstinence. Eur J Pharmacol 70:565-570. 